\title{
Preditores de infecções relacionadas à assistência à saúde em cirurgia cardíaca*
}

\author{
Predictors of healthcare associated infections in heart surgery
}

Predictores de infecciones relacionadas a la atención de salud en cirugía cardiaca

Érica Vieira de Andrade ${ }^{1}$, Maria Helena Barbosa² ${ }^{2}$ Ana Lúcia de Mattia ${ }^{3}$

\begin{abstract}
* Artigo extraído da dissertação de Mestrado "Análise da ocorrência de infecções hospitalares em pacientes submetidos à cirurgia cardíaca", defendida em 2011, vinculada ao Programa de Pós-Graduação em Atenção à Saúde da Universidade Federal do Triângulo Mineiro.

${ }^{1}$ Enfermeira, Mestre em Atenção à Saúde. Uberaba, MG, Brasil. E-mail: erica.vieira.andrade@terra.com.br.

${ }^{2}$ Enfermeira, Doutora em Enfermagem na Saúde do Adulto. Professora Adjunta da Universidade Federal do Triângulo Mineiro. Uberaba, MG, Brasil. Email: mhelena331@hotmail.com.

${ }^{3}$ Enfermeira, Doutora em Enfermagem. Professora Adjunta da Escola de Enfermagem da Universidade Federal de Minas Gerais. Belo Horizonte, MG, Brasil. E-mail: almattia@uol.com.br.
\end{abstract}

\section{RESUMO}

Esta pesquisa objetivou determinar a incidência de pacientes com infecções relacionadas à assistência à saúde e a localização topográfica destas infecções em pós-operatório de cirurgia cardíaca e identificar os fatores de risco associados à ocorrência destas infecções. Estudo retrospectivo com 460 pacientes submetidos à cirurgia cardíaca em um hospital de ensino de Minas Gerais. Os dados foram coletados das fichas de notificação de infecção hospitalar e dos prontuários. A incidência de pacientes com infecções relacionadas à assistência à saúde foi de $24,3 \%$ e a infecção do trato respiratório apresentou maior incidência (20,6\%). Tempo de intubação, tempo de permanência do cateter venoso central e tempo de permanência da sonda vesical de demora foram os preditores independentes. Os achados apontam para a necessidade de implementação de protocolos de cuidado e manutenção destes dispositivos invasivos para a prevenção e o controle das infecções relacionadas à assistência à saúde em cirurgia cardíaca.

Descritores: Infecção Hospitalar; Procedimentos Cirúrgicos Cardíacos; Enfermagem Perioperatória.

\section{ABSTRACT}

The objective of this study was to determine the incidence of patients with healthcare associated infections, the topographic location of those infections in the heart surgery postoperative period, and identify the risk factors associated with the occurrence of those infections. This retrospective study was performed with 460 heart surgery patients of a teaching hospital in Minas Gerais state. The data were collected from the hospital infection report forms and patients' records. The incidence of patients with healthcare associated infections was $24.3 \%$ and respiratory tract infection had the highest rates (20.6\%). Intubation time, permanence time of central venous catheter and indwelling urinary catheter were independent predictors. The findings point at the need to implement protocols for the care and maintenance of these invasive devices with the purpose of preventing and controlling healthcare associated infection in heart surgery.

Descriptors: Cross Infection; Cardiac Surgical Procedures; Perioperative Nursing.

\section{RESUMEN}

Se objetivó determinar la incidencia de pacientes con infecciones relacionadas a la atención de salud y la localización topográfica de estas infecciones en postoperatorio de cirugía cardíaca, e identificar los factores de riesgo asociados a la ocurrencia de tales infecciones. Estudio retrospectivo con 460 pacientes sometidos a cirugía cardíaca en hospital de enseñanza de Minas Gerais. Datos recolectados de fichas de notificación de infección hospitalaria y de historias clínicas. La incidencia de pacientes con infecciones relacionadas a la atención de salud fue de $24,3 \%$, la infección del tracto respiratorio presentó mayor incidencia (20,6\%). Tiempo de intubación, tiempo de permanencia del catéter venoso central y tiempo de permanencia de sonda vesical de demora fueron los predictores independientes. Los hallazgos orientan a la necesidad de implementación de protocolos de cuidado y mantenimiento de estos dispositivos invasivos para prevenir y controlar las infecciones relacionadas a la atención de salud en cirugía cardíaca.

Descriptores: Infección Hospitalaria; Procedimientos Quirúrgicos Cardíacos; Enfermería Perioperatoria. 


\section{INTRODUÇÃO}

Com o aumento das doenças cardiovasculares, a cirurgia cardíaca tornou-se mais frequente, sendo um procedimento complexo que engloba vários fatores de risco para a ocorrência de Infecções Relacionadas à Assistência à Saúde (IRAS) no período pós-operatório.

Como tais infecções ainda são um relevante problema de saúde pública, a Agência Nacional de Vigilância Sanitária vem desenvolvendo estratégias para melhorar a segurança do paciente nos serviços de saúde e o principal objetivo destas ações é reduzir os casos de IRAS no país. Estas iniciativas fundamentam-se em dois desafios globais lançados pela Organização Mundial de Saúde, cujos focos estão nas ações de melhoria da higienização das mãos em serviços de saúde e nas ações de melhoria dos procedimentos cirúrgicos(1).

Em cirurgias cardíacas, as infecções representam expressivas complicações pós-operatórias relacionadas a diversos fatores ${ }^{(2-3)}$ e no período perioperatório, o diagnóstico de enfermagem "risco para infecção" é um dos mais frequentes nos pacientes submetidos a estas cirurgias (4).

Nos últimos anos, observou-se uma mudança no perfil dos pacientes que se submetem a cirurgias cardíacas devido ao aprimoramento dos procedimentos diagnósticos e terapêuticos ${ }^{(5)}$. Muitos destes pacientes apresentam associação de comorbidades, aspecto relacionado ao envelhecimento populacional, o que aumenta o risco e a gravidade das IRAS no período pósoperatório(6). Além disso, os pacientes que realizam procedimentos cirúrgicos cardíacos ficam internados na Unidade de Terapia Intensiva (UTI) durante os primeiros dias e sabe-se que nesta unidade, as taxas de infecção e de multirresistência aos antimicrobianos são elevadas( ${ }^{(7)}$.

Embora vários preditores de IRAS no pós-operatório de cirurgia cardíaca tenham sido investigados, tais fatores ainda permanecem em discussão no meio científico(8-9). Ressalta-se, pois, que a identificação dos preditores de risco e sua relação com os desfechos pós-operatórios, principalmente com o risco de infecção, são fundamentais para a implementação de medidas preventivas, o que melhora o prognóstico e diminui os custos. Desta forma, conhecer os preditores locais torna-se essencial para um melhor planejamento da assistência( ${ }^{(8)}$.

Frente ao exposto, este estudo teve como objetivos determinar a incidência de pacientes com infecções relacionadas à assistência à saúde e a localização topográfica destas infecções em pós-operatório de cirurgia cardíaca e identificar os fatores de risco relacionados ao paciente, ao procedimento cirúrgico e ao perioperatório, associados à ocorrência de infecções em pacientes submetidos à cirurgia cardíaca.

\section{MÉTODOS}

Trata-se de um estudo retrospectivo, tipo coorte histórica, com abordagem quantitativa, realizado no Hospital de Clínicas da Universidade Federal do Triângulo Mineiro (HC/UFTM), um hospital de ensino e de grande porte localizado em Uberaba, Minas Gerais.

Este estudo avaliou os pacientes submetidos à cirurgia cardíaca no período de julho de 2005 a julho de 2010. Neste período, 589 pacientes realizaram cirurgia cardíaca, dos quais 460 atenderam aos critérios de inclusão e exclusão, constituindo-se na população (n) deste estudo. Foram incluídos nesta pesquisa os pacientes que se submeteram à cirurgia cardíaca com idade igual ou superior a 18 anos e foram excluídos os pacientes cujos números de prontuário não foram identificados, cujos prontuários não foram localizados e aqueles que foram a óbito durante o intra-operatório ou em até 48 horas de pós-operatório.

Os dados foram coletados das fichas de notificação de infecção hospitalar disponibilizadas pela Comissão de Controle de Infecção Hospitalar (CCIH) do HC/UFTM, e dos prontuários dos pacientes submetidos à cirurgia cardíaca. O acesso aos prontuários deu-se pelo fornecimento de seus respectivos números pelo Serviço de Cirurgia Cardíaca do hospital e posterior consulta no Serviço de Arquivo Médico.

A coleta dos dados iniciou-se após a aprovação do projeto de pesquisa pelo Comitê de Ética em Pesquisa da UFTM, sob parecer $n^{\circ} 1611 / 2010$, em conformidade com a Resolução 196/96 do Conselho Nacional de Saúde, sobre pesquisas envolvendo seres humanos. O Termo de Consentimento Livre e Esclarecido não foi utilizado, considerando que os dados foram obtidos dos prontuários e das fichas de notificação de infecção hospitalar dos pacientes e muitos deles residiam em outras cidades ou foram a óbito, o que tornaria inviável a assinatura do Termo. Ressalta-se que foi garantido o sigilo quanto à identificação dos sujeitos da pesquisa por meio da numeração dos instrumentos de coleta de dados. 
Para a coleta dos dados foi utilizado um instrumento (formulário) submetido à avaliação de conteúdo por cinco especialistas. Este instrumento englobava a investigação das seguintes variáveis: sociodemográficas e clínicas dos pacientes (idade, sexo, comorbidades, cirurgia cardíaca prévia) e tipo de cirurgia, classificação da cirurgia (eletiva, urgência ou emergência), uso de circulação extracorpórea (CEC), duração da cirurgia, tempo de intubação, tempo de permanência do cateter venoso central (CVC), tempo de permanência da sonda vesical de demora (SVD), ocorrência e localização topográfica das IRAS. Salienta-se que, nesta pesquisa, foram consideradas as infecções tanto notificadas pela $\mathrm{CClH}$, quanto aquelas diagnosticadas e registradas no prontuário pela equipe médica responsável pelo paciente.

Os dados coletados foram inseridos em uma planilha eletrônica do programa Excel® por meio de dupla entrada e após a validação, o banco de dados foi exportado para o programa estatístico Statistical Package for the Social Sciences (SPSS) versão 17.0 para proceder-se a análise. As variáveis qualitativas foram analisadas segundo estatística descritiva por meio da distribuição de frequência absoluta e percentual, enquanto para as variáveis quantitativas foram utilizadas as medidas descritivas de centralidade (média) e de dispersão (desvio padrão, valor mínimo e valor máximo).

Para identificar os fatores de risco associados à ocorrência de IRAS realizou-se, inicialmente, a análise bivariada utilizando-se o teste qui-quadrado e as medidas de associação (risco relativo e razão de chances). Foram consideradas com associação estatisticamente significativa, as variáveis com valor de $p<0,05$. Posteriormente, realizou-se a análise multivariada por meio da regressão logística. Para isto foram consideradas as variáveis que apresentaram na análise bivariada valor de $p<0,1$ e que também possuíam maior relevância clínica frente às demais variáveis. No modelo multivariado o nível de significância adotado foi de 0,05. Ressalta-se que para proceder à análise bivariada e multivariada, todas as variáveis estudadas foram dicotomizadas.

\section{RESULTADOS}

Na população estudada a maioria dos pacientes era do sexo masculino $280(60,9 \%)$ e a média de idade da população foi de 55,6 anos ( $\pm 12,9$ ), com variação de 18 a 81 anos, sendo a faixa etária de 50 a 59 anos a prevalente com $146(31,7 \%)$ pacientes. Quanto às comorbidades, a maioria 325 (70,6\%) dos pacientes apresentava hipertensão arterial sistêmica (HAS), mas o diabetes mellitus (DM) também foi frequente, presente em 105 $(22,8 \%)$ pacientes.

Quanto ao procedimento cirúrgico, observou-se que a cirurgia de revascularização do miocárdio isolada (RM) foi prevalente, sendo realizada em 218 (47,4\%) pacientes. As trocas valvares, mitral e aórtica, também obtiveram frequência expressiva, realizadas em $83(18,0 \%)$ e 69 $(15,0 \%)$ pacientes, respectivamente.

A maioria 452 (98,3\%) das cirurgias foi eletiva e apenas oito $(1,7 \%)$ foram classificadas como urgência. Ressalta-se que na população estudada não houve cirurgia classificada como emergência. Em relação ao uso da CEC, a maioria 449 (97,6\%) dos procedimentos cirúrgicos realizou-se com a utilização deste mecanismo, enquanto 11 (2,4\%) cirurgias foram realizadas sem CEC.

Quanto à ocorrência de IRAS, verificou-se que dos 460 pacientes participantes desta pesquisa, 112 adquiriram infecção durante a internação pós-operatória, o que resultou em uma incidência de pacientes com IRAS de $24,3 \%$. O número de sítios infecciosos por paciente variou de um a cinco, sendo que a maioria 86 (76,8\%) dos pacientes desenvolveu infecção em apenas um sítio corpóreo.

Quanto à localização topográfica, observou-se na população desta pesquisa, a ocorrência de IRAS em oito sítios corpóreos diferentes, sendo trato respiratório (20,6\%), sítio cirúrgico $(7,8 \%)$, trato urinário (2,2\%), corrente sanguínea (1,5\%), cutânea (em úlcera por pressão) $(0,9 \%)$, peritonite $(0,2 \%)$, endocardite $(0,2 \%)$ e otite $(0,2 \%)$.

Ocorreram, no total, 155 IRAS no pós-operatório, o que resultou em uma média de 1,4 infecções por paciente infectado. Destaca-se que na população estudada, 10 (2,2\%) pacientes evoluíram com sepse.

Em relação aos fatores de risco para a ocorrência de IRAS verificou-se que, na análise bivariada, as variáveis grupo etário, DM, doença pulmonar obstrutiva crônica (DPOC), cirurgia cardíaca prévia, duração da cirurgia, tempo de intubação, tempo de permanência do CVC e tempo de permanência da SVD apresentaram associação estatisticamente significativa $(p<0,05)$ (Tabela 1$)$.

Quanto ao grupo etário, os pacientes idosos apresentaram incidência de IRAS de 30,3\%. De acordo 
com o risco relativo, os idosos têm $49 \%$ a mais de risco de desenvolverem IRAS do que os adultos. A média de idade dos pacientes com infecção foi de 60,0 anos $( \pm 11,3)$ com variação de 22 a 80 anos. Entre os pacientes que desenvolveram IRAS no período pós-operatório verificou- se o tempo médio de duração da cirurgia de 4,3 horas ( \pm 1,1) com variação de 1,9 a 7,3 horas. O tempo de permanência do CVC e o tempo de permanência da SVD foram as variáveis que apresentaram maior risco relativo, respectivamente 6,36 e 8,56 (Tabela 1 ).

Tabela 1: Análise bivariada entre as variáveis relacionadas ao paciente, à cirurgia e ao perioperatório e a ocorrência de IRAS. Uberaba, MG, Brasil, 2005-2010.

\begin{tabular}{|c|c|c|c|c|c|c|c|}
\hline \multirow{3}{*}{ Variáveis } & \multicolumn{4}{|c|}{ IRAS } & \multirow{3}{*}{$\mathbf{R R}(\mathrm{IC})^{*}$} & \multirow{3}{*}{$\mathrm{RC}(\mathrm{IC})^{\star *}$} & \multirow{3}{*}{$\boldsymbol{P}^{* * *}$} \\
\hline & \multicolumn{2}{|c|}{ Sim } & \multicolumn{2}{|c|}{ Não } & & & \\
\hline & $\mathbf{n}$ & $\%$ & $\mathbf{n}$ & $\%$ & & & \\
\hline \multicolumn{8}{|l|}{ Grupo etário } \\
\hline Idoso & 56 & 30,3 & 129 & 69,7 & \multirow{2}{*}{$1,49(1,08-2,05)$} & \multirow{2}{*}{$1,70(1,10-2,61)$} & \multirow{2}{*}{0,015} \\
\hline Adulto & 56 & 20,4 & 219 & 79,6 & & & \\
\hline \multicolumn{8}{|l|}{ DM } \\
\hline Sim & 38 & 36,2 & 67 & 63,8 & \multirow{2}{*}{$1,74(1,25-2,40)$} & \multirow{2}{*}{$2,15(1,34-3,46)$} & \multirow{2}{*}{0,001} \\
\hline Não & 74 & 20,8 & 281 & 79,2 & & & \\
\hline \multicolumn{8}{|l|}{ DPOC } \\
\hline Sim & 27 & 51,9 & 25 & 48,1 & \multirow{2}{*}{$2,49(1,80-3,44)$} & \multirow{2}{*}{$4,10(2,27-7,43)$} & \multirow{2}{*}{$<0,001$} \\
\hline Não & 85 & 20,8 & 323 & 79,2 & & & \\
\hline \multicolumn{8}{|c|}{ Cirurgia cardíaca prévia } \\
\hline Sim & 16 & 39,0 & 25 & 61,0 & \multirow{2}{*}{$1,70(1,12-2,59)$} & \multirow{2}{*}{$2,15(1,10-4,20)$} & \multirow{2}{*}{0,022} \\
\hline Não & 96 & 22,9 & 323 & 77,1 & & & \\
\hline \multicolumn{8}{|l|}{ Duração da cirurgia } \\
\hline 4 horas ou mais & 66 & 34,4 & 126 & 65,6 & \multirow{2}{*}{$2,00(1,44-2,78)$} & \multirow{2}{*}{$2,53(1,64-3,91)$} & \multirow{2}{*}{$<0,001$} \\
\hline Menos que $4 \mathrm{~h}$ & 46 & 17,2 & 222 & 82,8 & & & \\
\hline \multicolumn{8}{|l|}{ Tempo de intubação } \\
\hline $24 \mathrm{~h}$ ou mais & 67 & 44,7 & 83 & 55,3 & \multirow{2}{*}{$3,08(2,23-4,25)$} & \multirow{2}{*}{$4,75(3,03-7,46)$} & \multirow{2}{*}{$<0,001$} \\
\hline Menos que $24 \mathrm{~h}$ & 45 & 14,5 & 265 & 85,5 & & & \\
\hline \multicolumn{8}{|l|}{ Permanência do CVC } \\
\hline 6 dias ou mais & 81 & 60,4 & 53 & 39,6 & & & \\
\hline Menos que 6 dias & 31 & 9,5 & 295 & 90,5 & $6,36(4,43-9,13)$ & $14,54(8,76-24,14)$ & $<0,001$ \\
\hline Permanência da SVD & & & & & & & \\
\hline 6 dias ou mais & 87 & 65,4 & 46 & 34,6 & $856(576-1272)$ & & $<0001$ \\
\hline Menos que 6 dias & 25 & 7,6 & 302 & 92,4 & $8,56(5,16-12,12)$ & $22,85(13,28-39,29)$ & $<0,001$ \\
\hline
\end{tabular}

Na análise multivariada por regressão logística, o tempo de intubação, o tempo de permanência do CVC e o tempo de permanência da SVD continuaram a apresentar associação estatisticamente significativa $(p<0,05)$ com a ocorrência de IRAS, sendo considerados os fatores de risco independentes para este desfecho (Tabela 2). 
Tabela 2: Regressão logística dos fatores associados à ocorrência de IRAS nos pacientes submetidos à cirurgia cardíaca. Uberaba, MG, Brasil, 2005-2010.

\begin{tabular}{|c|c|c|c|c|c|c|}
\hline \multirow{3}{*}{ Variáveis } & \multicolumn{4}{|c|}{ IRAS } & \multirow{3}{*}{ RC (IC) } & \multirow{3}{*}{$p$} \\
\hline & \multicolumn{2}{|c|}{ Sim } & \multicolumn{2}{|c|}{ Não } & & \\
\hline & $\mathbf{n}$ & $\%$ & $\mathbf{n}$ & $\%$ & & \\
\hline \multicolumn{7}{|l|}{ Grupo etário } \\
\hline Idoso & 56 & 30,3 & 129 & 69,7 & \multirow{2}{*}{$0,73(0,39-1,34)$} & \multirow{2}{*}{0,307} \\
\hline Adulto & 56 & 20,4 & 219 & 79,6 & & \\
\hline \multicolumn{7}{|l|}{ DM } \\
\hline Sim & 38 & 36,2 & 67 & 63,8 & \multirow{2}{*}{$1,48(0,77-2,82)$} & \multirow{2}{*}{0,238} \\
\hline Não & 74 & 20,8 & 281 & 79,2 & & \\
\hline \multicolumn{7}{|l|}{ DPOC } \\
\hline Sim & 27 & 51,9 & 25 & 48,1 & \multirow{2}{*}{$2,21(0,99-4,97)$} & \multirow{2}{*}{0,054} \\
\hline Não & 85 & 20,8 & 323 & 79,2 & & \\
\hline \multicolumn{7}{|l|}{ Cirurgia cardíaca prévia } \\
\hline Sim & 16 & 39,0 & 25 & 61,0 & \multirow{2}{*}{$1,74(0,70-4,32)$} & \multirow[b]{2}{*}{0,230} \\
\hline Não & 96 & 22,9 & 323 & 77,1 & & \\
\hline \multicolumn{7}{|l|}{ Duração da cirurgia } \\
\hline 4 horas ou mais & 66 & 34,4 & 126 & 65,6 & \multirow{2}{*}{$1,50(0,84-2,69)$} & \multirow{2}{*}{0,170} \\
\hline Menos que 4 horas & 46 & 17,2 & 222 & 82,8 & & \\
\hline \multicolumn{7}{|l|}{ Tempo de intubação } \\
\hline $24 \mathrm{~h}$ ou mais & 67 & 44,7 & 83 & 55,3 & \multirow{2}{*}{$2,34(1,29-4,26)$} & \multirow{2}{*}{0,005} \\
\hline Menos que $24 \mathrm{~h}$ & 45 & 14,5 & 265 & 85,5 & & \\
\hline \multicolumn{7}{|l|}{ Permanência do CVC } \\
\hline 6 dias ou mais & 81 & 60,4 & 53 & 39,6 & \multirow{3}{*}{$4,00(2,07-7,72)$} & \multirow{2}{*}{$<0,001$} \\
\hline Menos que 6 dias & 31 & 9,5 & 295 & 90,5 & & \\
\hline \multicolumn{6}{|l|}{ Permanência da SVD } & \\
\hline 6 dias ou mais & 87 & 65,4 & 46 & 34,6 & \multirow{2}{*}{$7,22(3,61-14,45)$} & \multirow{2}{*}{$<0,001$} \\
\hline Menos que 6 dias & 25 & 7,6 & 302 & 92,4 & & \\
\hline
\end{tabular}

O tempo médio de intubação dos pacientes com IRAS foi de 55,8 horas $( \pm 78,2$ ) com variação de cinco a 469 horas e o tempo médio dos pacientes sem infecção foi de 16,6 horas ( $\pm 14,8$ ) com variação de quatro a 194 horas.

Em relação à permanência do CVC, o tempo médio entre os pacientes que desenvolveram infecção foi de 14,2 dias $( \pm 13,6)$ com variação de um a 71 dias, enquanto entre os pacientes sem infecção o tempo médio foi de quatro dias $( \pm 2,6)$ com variação de um a 28 dias. Quanto à SVD, os pacientes com IRAS apresentaram tempo médio de permanência de 14,7 dias $( \pm 12,5)$ com variação de dois a 68 dias e os pacientes que não adquiriram infecção tiveram tempo médio de 3,6 dias $( \pm 2,1)$ com variação de um a 18 dias.

\section{DISCUSSÃO}

No presente estudo, a maioria dos pacientes era do sexo masculino, o que também foi evidenciado por outros autores $^{(10-11)}$. A população estudada apresentou média de idade de 55,6 anos, resultado semelhante ao de outro

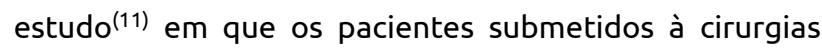
cardíacas apresentaram média de idade de 57,8 anos. Entretanto, outra pesquisa apresentou população mais envelhecida ${ }^{(3)}$. Isto aponta para a necessidade de políticas públicas de prevenção e controle dos fatores de risco cardiovasculares voltadas para a população mais jovem, considerando a relevância dos aspectos socioambientais e hábitos de vida desta população.

No que se refere às comorbidades, a maioria da população deste estudo apresentava HAS e embora com menor frequência, o número de pacientes com DM também foi expressivo. Em outras investigações estas comorbidades mostraram-se, do mesmo modo, com elevada frequência entre os sujeitos avaliados ${ }^{(12-14)}$.

Observou-se que das cirurgias cardíacas realizadas, a RM foi predominante ocorrendo em $47,4 \%$ dos pacientes, resultado semelhante ao encontrado em outras pesquisas que também evidenciaram a maior frequência desta cirurgia quando comparada às demais ${ }^{(2,15)}$. Em outra investigação as trocas valvares representaram a maioria das cirurgias ${ }^{(3)}$.

Em relação à ocorrência de IRAS no pós-operatório de cirurgias cardíacas, verificou-se neste estudo que a incidência de pacientes com infecção foi de $24,3 \%$. Este resultado corrobora os dados da literatura, os quais demonstram uma incidência variável que abrange de 5,0\% a $26,5 \%$, referindo-se a pesquisas realizadas não somente no Brasil, como em vários países (2,8,12,15-16). 
Observou-se, na população estudada, a ocorrência de IRAS em oito sítios corpóreos diferentes, sendo que a infecção do trato respiratório apresentou maior incidência, o que também foi evidenciado por outros autores ${ }^{(2,17)}$. Apesar dos avanços tecnológicos dos últimos tempos nas cirurgias cardíacas, os resultados mostram que ainda existem muitos desafios a serem superados, considerando que outras realidades também se defrontam com um número elevado de infecção do trato respiratório no pós-operatório nessa especialidade. A intubação traqueal e a dor são eventos relevantes para ocorrência desta complicação.

Verificou-se, no presente trabalho, que o sítio cirúrgico correspondeu ao segundo local topográfico mais frequente na ocorrência das IRAS, o que diverge de outras pesquisas em que a infecção da ferida operatória, seja esternal e/ou na região da safenectomia, representou o sítio infeccioso de maior incidência(12,15-16).

Neste trabalho, $76,8 \%$ dos pacientes com IRAS adquiriram infecção em apenas um sítio corpóreo, o que corrobora outros estudos em que mais de $70,0 \%$ dos pacientes infectados desenvolveram uma única infecção ${ }^{(2,16)}$.

Referente aos fatores de risco para a ocorrência de IRAS após cirurgias cardíacas verificou-se, neste estudo, que o tempo de intubação, o tempo de permanência do CVC e o tempo de permanência da SVD foram os preditores independentes para o desfecho infecção. Ressalta-se, a partir destes resultados, a importância dos procedimentos e artigos invasivos no desenvolvimento de IRAS, apontando para a necessidade de maior atenção e cuidado no uso destes dispositivos. A equipe multiprofissional de saúde deve estar capacitada para prevenir e controlar eventos adversos desde a instalação até a retirada destes dispositivos, com foco em sua manutenção, visando minimizar os riscos e garantir uma assistência de qualidade. Outro aspecto que merece destaque refere-se aos tipos de dispositivos invasivos adotados pelas instituições. Isto exige estratégias como, por exemplo, a implantação de um serviço de controle de qualidade dos artigos médico-hospitalares. A assistência intensiva utiliza vários dispositivos invasivos para que o cuidado adequado seja prestado ao paciente, entretanto estes recursos podem causar complicações, das quais se destaca a infecção ${ }^{(18)}$. Em pesquisa realizada na Sala de Emergência da Unidade de Pronto Atendimento de um hospital universitário, dos pacientes que adquiriram infecções relacionadas ao cuidar em saúde, $93,1 \%$ utilizaram procedimentos invasivos ${ }^{(19)}$.

Assim como neste estudo, outras pesquisas também evidenciaram o tempo de intubação como preditor independente de IRAS, principalmente de pneumonia ${ }^{(2-3)}$. Outros autores destacaram as vantagens da redução do tempo de intubação após cirurgias cardíacas, pois os pacientes extubados ainda na sala de operação, bem como aqueles que foram extubados em menos de quatro horas da intubação apresentaram recuperação mais rápida, menor tempo de permanência na UTI e no hospital e menos episódios de reintubação, indicando que a extubação precoce pode representar uma boa relação custo-benefício(20-21).

Quanto ao tempo de permanência do CVC, estudos anteriores também identificaram esta variável como preditora para IRAS ${ }^{(8,22)}$. Outros autores observaram em sua pesquisa as graves consequências das infecções associadas ao CVC. Neste trabalho realizado em uma UTI de Porto Alegre, 37 sujeitos desenvolveram 43 infecções relacionadas ao cateter, sendo que cinco pacientes apresentaram sepse associada ao dispositivo. Dos 37 pacientes, 21 foram a óbito durante a internação hospitalar, 12 deles por septicemia. Ainda segundo os pesquisadores, é fundamental uma alta suspeita clínica para que a infecção seja diagnosticada corretamente e iniciada a conduta terapêutica apropriada ${ }^{(23)}$.

Em relação ao tempo de permanência da SVD, o presente estudo corrobora outros trabalhos que identificaram esta variável como fator de risco para infecção, principalmente do trato urinário(24-25). Observou-se, em outra investigação, que $68,4 \%$ dos pacientes com infecção do trato urinário permaneceram com o cateter vesical por mais de seis dias, sendo o tempo de cateterização juntamente com o tempo de internação os fatores de risco identificados para a referida infecção(25).

As evidências desta pesquisa explicitam a importância dos fatores extrínsecos ao paciente como preditores de infecções após cirurgias cardíacas, cabendo então destacar o papel essencial da equipe de saúde na prevenção destas complicações, aderindo-se totalmente às práticas assistenciais seguras recomendadas. A Prática Baseada em Evidência, a partir dos protocolos já 
existentes, é a ferramenta essencial para prevenção e controle das IRAS nesta população.

\section{CONCLUSÃO}

Neste estudo, a incidência de pacientes com IRAS foi de $24,3 \%$ e observou-se a ocorrência de infecção em oito sítios corpóreos diferentes, sendo que a do trato respiratório apresentou maior incidência. O tempo de intubação, o tempo de permanência do cateter venoso central e o tempo de permanência da sonda vesical de demora foram os fatores de risco independentemente associados à ocorrência de infecção pós-operatória na população estudada.

Os resultados desta pesquisa indicam que há necessidade de ampliar a vigilância ativa de IRAS após cirurgias cardíacas, bem como implementar estratégias para prevenção e controle destas complicações. As estratégias devem abranger o treinamento e a capacitação da equipe multiprofissional acerca da utilização de dispositivos invasivos, além da implantação e avaliação dos protocolos institucionais de assistência e dos indicadores de segurança e qualidade em pós-

\section{REFERÊNCIAS}

1. Carcute $D$. Anvisa apresenta estratégias para segurança do paciente em hospitais e clínicas [Internet]. Brasília: Agência Nacional de Vigilância Sanitária; 2010 Apr 09 [cited 2010 Apr 10]. Available from: http://s.anvisa.gov.br/wps/s/r/pc. 2. De Santo LS, Bancone C, Santarpino G, Romano G, De Feo M, Scardone $M$, et al. Microbiologically documented nosocomial infections after cardiac surgery: an 18-month prospective tertiary care centre report. Eur J Cardiothorac Surg. 2008;33(4):666-72.

3. Hortal J, Giannella M, Pérez MJ, Barrio JM, Desco M, Bouza E, et al. Incidence and risk factors for ventilator-associated pneumonia after major heart surgery. Intensive Care Med. 2009;35(9):1518-25.

4. Galdeano LE, Rossi LA, Santos CB, Dantas RAS. Diagnósticos de enfermagem no perioperatório de cirurgia cardíaca. Rev Esc Enferm USP. 2006;40(1):26-33.

5. Strabelli TMV, Stolf NAG, Uip DE. Uso prático de um índice de risco de complicações após cirurgia cardíaca. Arq Bras Cardiol. 2008;91(5):342-7.

6. Gelape CL. Infecção do sítio operatório em cirurgia cardíaca. Arq Bras Cardiol. 2007;89(1):e3-e9.

7. Lima ME, Andrade D, Haas VJ. Avaliação prospectiva da ocorrência de infecção em pacientes críticos de Unidade de Terapia Intensiva. Rev Bras Ter Intensiva. 2007;19(3):342-7. 8. Ledur P, Almeida L, Pellanda LC, Schaan BD. Preditores de infecção no pós-operatório de cirurgia de revascularização miocárdica. Rev Bras Cir Cardiovasc. 2011;26(2):190-6.

9. Lee YP, Feng MC, Wu LC, Chen SH, Chen YH, Chiu CC, et al. Outcome and risk factors associated with surgical site infections after cardiac surgery in a Taiwan medical center. J Microbiol Immunol Infect. 2010;43(5):378-85. operatório de cirurgia cardíaca. Todas essas ações devem estar norteadas pela Prática Baseada em Evidência.

Este estudo apresentou como limitação a impossibilidade de avaliação de resultados de exames laboratoriais dos pacientes e de aspectos relacionados ao instrumental cirúrgico e ao ambiente da sala de operação, decorrentes de falhas nos dados secundários utilizados como fonte de informação deste estudo. Entretanto, tais limitações não comprometeram os achados obtidos nesta investigação.

Esta pesquisa mostra-se relevante para a prática clínica visto que a identificação de preditores de risco permite prevenir de maneira eficaz a ocorrência de infecções nesta população. Além disso, os dados apresentados possibilitam o desenvolvimento de estudos prospectivos que tenham como objetivos, dentre outros, avaliar e comparar incidências de infecção, avaliar medidas preventivas e de intervenção relacionadas a um preditor específico, monitorar eventos adversos relacionados aos dispositivos invasivos e avaliar a adesão da equipe às práticas e aos procedimentos de segurança do cuidado.

10. Segers $P$, Speekenbrink RGH, Ubbink DT, van Ogtrop ML, Mol BA. Prevention of nosocomial infection in cardiac surgery by descontamination of the nasopharynx and oropharynx with chlorhexidine gluconate: a randomized controlled trial. JAMA. 2006;296(20):2460-6.

11. Sá MPBO, Silva DO, Lima ENS, Lima RC, Silva FPV, Rueda FG, et al. Mediastinite no pós-operatório de cirurgia cardiovascular: análise de 1038 cirurgias consecutivas. Rev Bras Cir Cardiovasc. 2010;25(1):19-24.

12. Blasco-Colmenares E, Perl TM, Guallar E, Baumgartner WA, Conte JV, Alejo D, et al. Aspirin plus clopidogrel and risk of infection after coronary artery bypass surgery. Arch Intern Med. 2009;169(8):788-96.

13. Guimarães RCM, Rabelo ER, Moraes MA, Azzolin K. Gravidade de pacientes em pós-operatório de cirurgia cardíaca: uma análise evolutiva segundo o TISS-28. Rev. Latino-Am. Enfermagem [Internet]. 2010 [cited 2011 Aug 02];18(1). Available from: http://www.scielo.br/pdf/rlae/v18n1/pt 10.pdf. 14. Fernandes MVB, Aliti G, Souza EN. Perfil de pacientes submetidos à cirurgia de revascularização miocárdica: implicações para o cuidado de enfermagem. Rev. Eletr. Enf. [Internet]. 2009 [cited 2010 Sep 22];11(4):993-9. Available from: http://www.fen.ufg.br/revista/v11/n4/v11n4a25.htm. 15. Nosrati M, Boroumand M, Tahmasebi S, Sotoudeh $M$, Sheikhfathollahi M, Goodarzynejad H. Excess costs associated with common healthcare-associated infections in an Iranian cardiac surgical unit. J Hosp Infect. 2010;76(4):304-7.

16. Roulia K, Apostolopoulou E. Surveillance of health care associated infections in patients after coronary artery bypass graft surgery. Review Clinical Pharmacology and Pharmacokinetics. 2010;24(1):25-30.

17. Chan RPC, Galas FRBG, Hajjar LA, Bello CN, Piccioni MA, Auler Jr JOC. Intensive perioperative glucose control does not 
improve outcomes of patients submitted to open-heart surgery: a randomized controlled trial. Clinics. 2009;64(1):51-60.

18. Diener JRC, Coutinho MSSA, Zoccoli CM. Infecções relacionadas ao cateter venoso central em terapia intensiva. Rev Assoc Med Bras. 1996;42(4):205-14.

19. Oliveira AC, Andrade FS, Diaz MEP, Iquiapaza RA. Colonização por micro-organismo resistente e infecção relacionada ao cuidar em saúde. Acta Paul Enferm. [Internet]. 2012 [cited 2012 July 08];25(2):183-9. Available from: http://www.scielo.br/pdf/ape/v25n2/a05v25n2.pdf. 20. Chamchad D, Horrow JC, Nachamchik L, Sutter FP, Samuels $L E$, Trace $C L$, et al. The impact of immediate extubation in the operating room after cardiac surgery on intensive care and hospital lengths of stay. J Cardiothorac Vasc Anesth. 2010;24(5):780-4.

21. Rashid A, Sattar KA, Dar MI, Khan AB. Analyzing the outcome of early versus prolonged extubation following cardiac surgery. Ann Thorac Cardiovasc Surg. 2008;14(4):218-23.

22. Mesiano ERAB, Merchán-Hamann E. Infecções da corrente sangüínea em pacientes em uso de cateter venoso central em unidades de terapia intensiva. Rev. Latino-Am. Enfermagem [Internet]. 2007 [cited 2011 Sep 14];15(3). Available from: http://www.scielo.br/pdf/rlae/v15n3/pt v15n3a14.pdf.

23. Marques Netto S, Echer IC, Kuplich NM, Kuchenbecker R, Kessler F. Infecção de cateter vascular central em pacientes adultos de um centro de terapia intensiva. Rev Gaúcha Enferm. 2009;30(3):429-36.

24. Kollef MH, Sharpless L, Vlasnik J, Pasque C, Murphy D, Fraser VJ. The impact of nosocomial infections on patient outcomes following cardiac surgery. Chest. 1997;112(3):666-75.

25. Stamm AMNF, Sakamoto KS, Cipriano ZM, Campos ML. Infecção do trato urinário relacionada à cateterização vesical: análise multivariada de fatores de risco em uma população geriátrica e não geriátrica. Rev Bras Med. 2007;64(1/2):18-25.

Artigo recebido em 29/11/2012.

Aprovado para publicação em 16/09/2013.

Artigo publicado em 31/12/2013. 